\title{
Ending Eclampsia: PHC PH/E_plus Model
}

Charlotte E. Warren

Population Council

Follow this and additional works at: https://knowledgecommons.popcouncil.org/departments_sbsr-rh

Part of the Demography, Population, and Ecology Commons, Family, Life Course, and Society Commons, International Public Health Commons, Maternal and Child Health Commons, and the Women's Health Commons How does access to this work benefit you? Let us know!

\section{Recommended Citation}

Warren, Charlotte E. 2018. "Ending Eclampsia: PHC PH/E_plus Model," Ending Eclampsia Research Brief. Washington, DC: Population Council. 

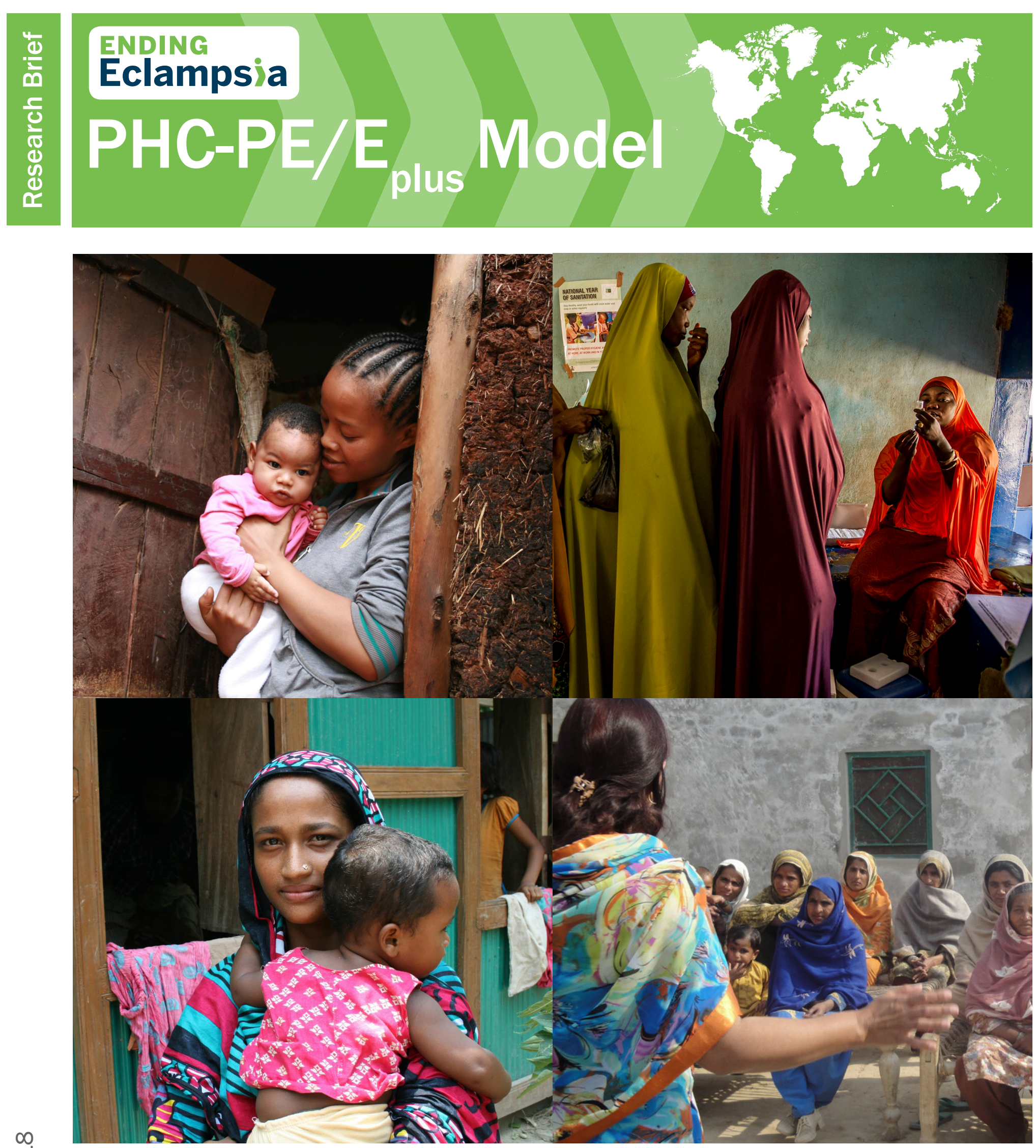

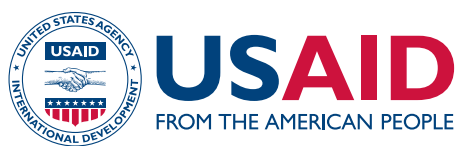

Made possible by the generous support of the American people through the United States

Agency for International Development (USAID).
The Population Council conducts research and delivers solutions that improve lives around the world. Big ideas supported by evidence: It's our model for global change. popcouncil.org

(c) 2018 The Population Council, Inc

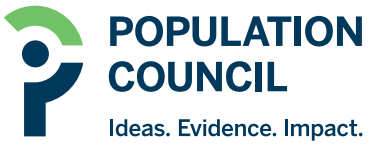




\section{THE PROBLEM}

Each day around the world, 830 women die from pregnancy- and childbirthrelated causes. In sub-Saharan Africa, 200,000 women die annually, and in Southern Asia 66,000 die each year. The second most common cause (after postpartum hemorrhage) is pre-eclampsia and eclampsia (PE/E)-lifethreatening, high blood pressure and excess protein in the urine after 20 weeks gestation. These deaths are preventable, yet essential medicines and tools to treat this disorder are often unavailable in low-resource settings.

$\mathrm{PE} / \mathrm{E}$ most often occurs during the second half of pregnancy, but can happen before, during, or after delivery. It can lead to seizures, kidney and liver damage, and death, and increases the risk of preterm births, low birth-weight, anemia, and stunting. Women who have PE/E have an increased risk of developing cardiovascular disease and other non-communicable diseases in later life.

The World Health Organization (WHO) recommends magnesium sulphate $\left(\mathrm{MgSO}_{4}\right)$ as the most effective, safe, and low-cost anticonvulsant treatment for severe pre-eclampsia and eclampsia. Twenty-eight countries include $\mathrm{MgSO}_{4}$ on their Essential Medicines List and have protocols on its use, but in practice, the drug is often unavailable at community facilities or is not used in compliance with guidelines.

\section{PHC PE/E CORE MODEL DEVELOPMENT}

Between 2008 and 2014, the Population Council implemented a twophase intervention, supported by the John D. and Catherine T. MacArthur Foundation, in Bangladesh, Mexico, and Nigeria. The first phase (2008-2011) involved training, mentoring, and supervising providers at secondary facilities on detection and management of PE/E patients. This included training on the administration of a loading dose of $\mathrm{MgSO}_{4}$. The providers then trained and mentored additional providers at their respective health facilities on the use of $\mathrm{MgSO}_{4}$.

The second phase of the intervention (2012-2014) focused on primary health care (PHC) facilities, specifically on training PHC providers to administer a modified loading dose of $\mathrm{MgSO}_{4}$ (intra-muscular only) and refer women to secondary health facilities for monitoring. PHC providers received continued technical support from trainings and routine mentorship.

The Council conducted an implementation research study to evaluate this task shifting process around the prevention, detection, and management of $\mathrm{PE} / \mathrm{E}$ at the PHC level, hereby defined as the PHC PE/E Core Model. The study showed that not only were PHC providers effectively able to carry out this task, but maternal mortality in the intervention group decreased.

These components: Training PHC providers on detection and management of $\mathrm{PE} / \mathrm{E}$, administration of a loading dose of $\mathrm{MgSO}_{4}$, referral of women to secondary facilities for monitoring, and reinforcing proper detection and management of $P E / E$ at secondary facilities are included in the PHC PE/E Core Model (Figure 1).

\section{$\mathrm{PE} / \mathrm{E}$ in Brief}

- Pre-eclampsia is a condition in pregnant women marked by an increase in blood pressure and protein in urine after 20 weeks gestation.

- Providing high quality antenatal care improves the prevention and early detection of pre-eclampsia and can prevent its progression to eclampsia.

- Eclampsia is a life-threatening condition characterized by convulsions in women with pre-eclampsia.

- Women in developing countries are 300 times more likely to die from eclampsia than women in developed countries.

- Prescribing low-dose aspirin and calcium to at-risk women can lower their risk of developing pre-eclampsia and eclampsia.

- Severe pre-eclampsia can be managed by administering anti-hypertensive drugs and magnesium sulphate $\left(\mathrm{MgSO}_{4}\right)$.

- $\mathrm{MgSO}_{4}$ is the safest and most effective treatment for severe $\mathrm{PE} / \mathrm{E}$.

- Pre-eclampsia and other hypertensive disorders in pregnancy increase the risk of preterm births, which can lead to low birth weight, anemia, and stunting.

- Improved prevention, increased detection, and effective treatment of $\mathrm{PE} / \mathrm{E}$ can prevent unnecessary maternal and newborn deaths.

The Ending Eclampsia project seeks to expand access to proven, underutilized interventions and commodities for the prevention, early detection, and treatment of pre-eclampsia and eclampsia and strengthen global partnerships. 


\section{SCALE UP OF PHC PE/E CORE MODEL}

The primary goal of the Ending Eclampsia project is to expand the use of $\mathrm{MgSO}_{4}$ and other underutilized commodities and strategies to prevent and treat preeclampsia and eclampsia (PE/E). Collaborating with national policymakers, ministries of health, community leaders, and health providers to increase access to highimpact, low-cost interventions.

The Ending Eclampsia project has been implementing the PHC PE/E Core Model in select regions in Bangladesh, Nigeria, and Pakistan. Replicating the core activities was crucial to demonstrate that the PHC PE/E Core Model is acceptable and feasible in varied settings. Prior to initiating implementation of the PHC PE/E Core Model, any organization--whether a ministry of health, an international non-governmental organization, or a local organization--must consider the reality on the ground within the particular context. Monitoring data and preliminary analyses across these three countries indicate that task shifting of oral antihypertensive drugs to PHCs is feasible and effective.
For the PHC PE/E Core Model to be the most effective (Figure 1), as with any complex health intervention, there is a certain health system context that must be in place. This includes:

- Supportive policy environment;

- Collaborative stakeholder engagement;

- Sustained funding for health;

- Operational infrastructure;

- Adequate and skilled human resources; and

- Functioning supply chain for essential drugs and commodities.

\section{IDENTIFYING “PLUS” MODULES}

Starting in 2016, Ending Eclampsia initiated six implementation research studies in three countries to identify and assess additional components that, in conjunction with the Core Model, can further improve prevention, detection, and management of $\mathrm{PE} / \mathrm{E}$.

FIGURE 1: PHC PE/E ${ }_{\text {plus }}$ Model

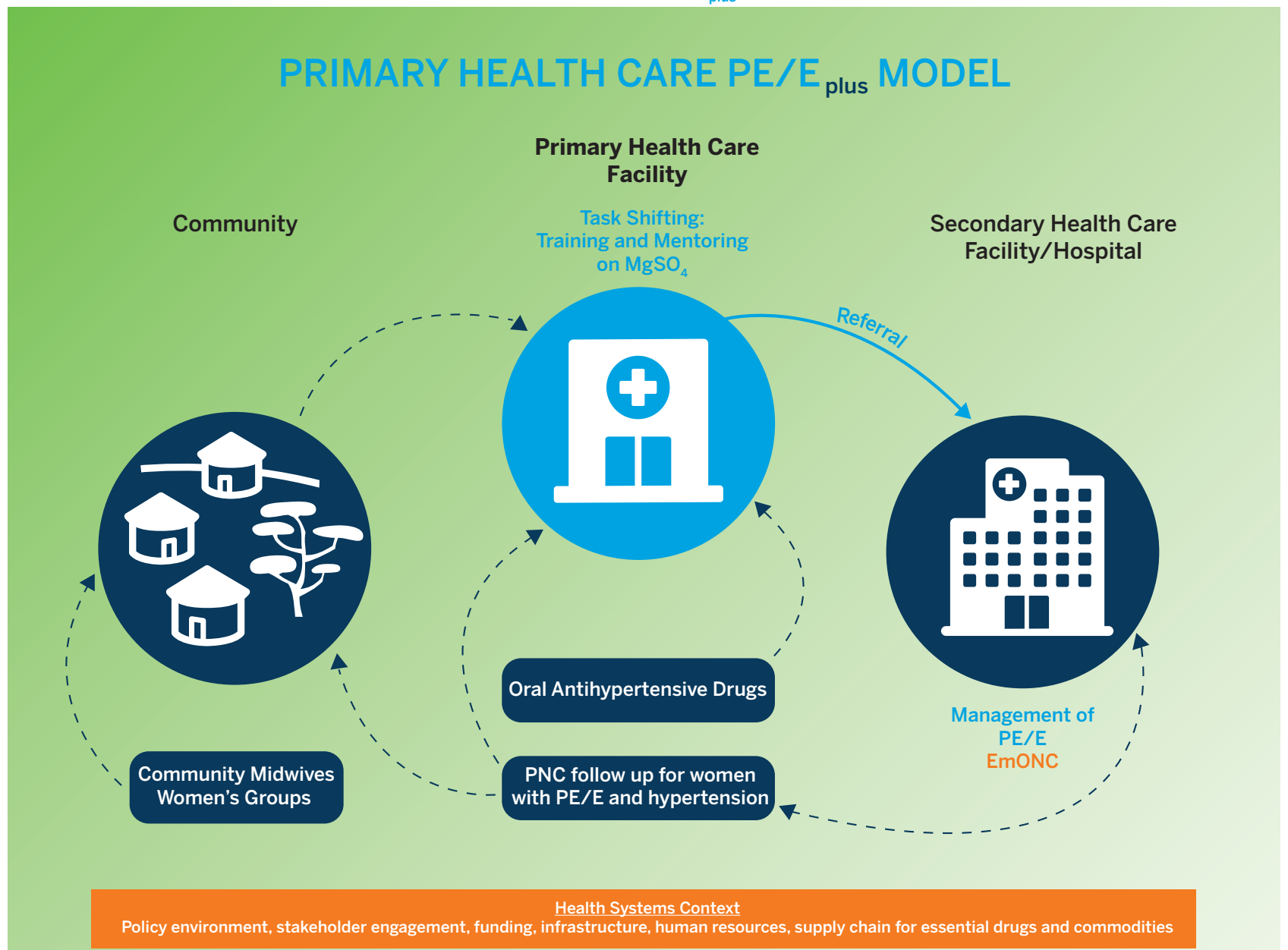




\section{Introducing antihypertensives at PHC}

In Nigeria and Bangladesh, Ending Eclampsia introduced an antihypertensive drug (oral methyldopa) at PHC level to improve management of hypertension in pregnancy with the ultimate goal of preventing progression to $\mathrm{PE} / \mathrm{E}$.

An antihypertensive drug module should be included, if possible, in efforts to improve prevention, detection, and management of PE/E (Figure 1). This module requires the availability of oral antihypertensive drugs that are safe for use in pregnancy (alpha methyldopa), training of PHC providers on the diagnosis of hypertension in pregnancy and administration of antihypertensive drugs, as well as how to provide adequate explanation of the condition, management protocols, and danger signs in pregnancy to women with high blood pressure.

\section{Engaging community women's groups (Nigeria)}

Women's group leaders were trained on health information that focused on antenatal care (ANC) and postnatal care (PNC) using a simplified pictorial job aid and log book to record activities.

Trained women's group leaders provide health education and information to their peers during their regular meetings. The expectation is that in doing so, more women will be empowered to use health facilities for themselves or to encourage their relatives for ANC/ PNC. In this way, ANC attendance, pre-eclampsia case detection and management, and quality of care for other services will improve.

Community groups with women, men, or a mix of the genders, can be targeted as channels for sharing important health information with communities (Figure 1). Clear, pictorial job aids can be used to support the group leaders in delivering key health messages about danger signs in pregnancy, specifically PE/E to the members of their group. Increased awareness of PE/E can potentially lead to a woman seeking care sooner than she would otherwise if she were to experience complications in pregnancy.

\section{Task-shifting to community midwives (Pakistan)}

Through implementation research conducted in Pakistan, researchers are assessing community midwives' (CMWs) ability to screen and detect PE/E cases, to provide a modified loading dose of $\mathrm{MgSO}_{4}$ to the appropriate clients, and to refer them for further management.
In addition, this study aims to enhance the collaboration between CMWs and local health workers (LHWs) by encouraging LHWs to refer pregnant women for group ANC sessions provided by local CMWs, and to improve ANC and PNC counselling skills of CMWs so as to enable them to manage postpartum hypertension.

\section{Explore postnatal outcomes}

About $25 \%$ of women with hypertensive disorders in pregnancy (HDPs), especially those with a severe condition, experience a deterioration of end organ functions during puerperium (period spanning $6-8$ weeks after delivery).In Bangladesh and Nigeria, Ending Eclampsia researched health outcomes of women and newborns after pregnancies complicated by HDPs and followed up with them at nine weeks, six months and one year after delivery to evaluate the nature of care they received, assess their health status, and identify any patterns in development of morbidities.

Trained nurses and midwives at the maternity unit conduct follow ups, which include reviewing treatment records from delivery onward, performing routine medical checkups, and surveying participants.

The importance of postnatal care is not often emphasized enough in any context. Women who experienced hypertension in pregnancy are particularly susceptible to ongoing complications or morbidities. Therefore, we recommend that PNC becomes a focus for essential care of women with a history of hypertension in pregnancy

(Figure 1.)

\section{NEXT STEPS}

Detailed results of the Ending Eclampsia project in each country will be available in 2019. Cross-country learnings will further refine the $\mathrm{PHC} \mathrm{PE} / \mathrm{E}_{\text {plus }}$ Model as a framework for global policy, program, and research strategies to address the problem of hypertension in pregnancy in a context-sensitive way. A package of step-wise recommendations around task-shifting will be compiled and disseminated for integration within country-level maternal health strategies. At regional and global levels, Ending Eclampsia plans to share the PHC PE/ $\mathrm{E}_{\text {plus }}$ model, related findings, and tools for adoption and scale up in varied settings.

Contact

Charlotte Warren, Senior Associate, Population Council cwarren@popcouncil.org

Salisu Mohammed Ishaku, Reproductive Health Program Director, Population Council sishaku@popcouncil.org

Suggested Citation

Charlotte Warren. 2018. "PHC PH/E $\mathrm{E}_{\text {lus }}$ Model," Ending Eclampsia Research Brief. Abuja: Population Council. 